Background: The journey of axial spondyloarthritis (axSpA) for most patients is slow and arduous.

Objectives: The goal of this analysis is to describe the journey to diagnosis and further management in axSpA patients.

Methods: 2,846 unselected patients participated in EMAS, a cross-sectional study (2017-2018) across 13 European countries. Descriptive analysis of sociodemographic factors, insurance scheme, diagnostic journey and post diagnosis healthcare utilization was performed. Mann-Whitey test was used to analyse possible differences between BASDAI ( $>4 \vee \leq 4)$ and the number of visits to healthcare professionals and follow-up tests undertaken.

Results: Mean age was 43.9 years, $61.3 \%$ were female, $48.1 \%$ university educated, $67.9 \%$ married, $53.9 \%$ employed and $81.7 \%$ had public health insurance. Mean age at symptoms onset was 26.6 (11.1), while mean age at diagnosis was 33.7 (11.5) and mean diagnostic delay was 7.4 years. Over $50 \%$ had a diagnostic delay of $\geq 4$ years. Prior to receiving a diagnosis, patients visited on average 2.6 specialists. The most commonly performed diagnostic tests were $\mathrm{x}$-rays (72.3\%), HLA B27 tests $(65.4 \%)$ and MRIs $(64.3 \%) .78 .4 \%$ were diagnosed by a rheumatologist while $14.9 \%$ received their diagnosis by a GP. Patients who experienced a diagnostic delay of more than a year $(n=2,208)$ undertook a considerable number of visits to specialists and medical tests in the year prior to participating in EMAS, which increased with disease activity. Patients with active disease (BASDAI $>4$ ) reported a higher number of visits to rheumatologists $(3.7 \pm 3.5$ vs $2.9 \pm 2.6)$, general practitioners $(6.6 \pm 10.0$ vs $3.5 \pm 4.1)$, physiotherapists $(19.3 \pm 25.0$ vs $11.7 \pm 17.0)$, and psychologists/psychiatrists $(3.4 \pm 10.7$ vs $1.9 \pm 7.7)$. Patients with active disease also undertook more $x$-rays $(1.8 \pm 2.8$ vs. $1.3 \pm 1.9)$, MRI scans $(0.9 \pm 1.2$ vs. $0.6 \pm 1.1)$, and blood tests $(4.7 \pm 4.4$ vs $3.6 \pm 3.2)$. However, one in five patients visited the rheumatologist only once in the year prior to EMAS (21.1\%).

Conclusion: Diagnostic delay continues to be a key challenge in the axSpA patient journey, with patients waiting an average of 7.4 years and visiting multiple doctors prior to diagnosis. Once diagnosed, disease management presents a further challenge, as patients with higher disease activity reported more healthcare professional visits as well as medical tests. Safeguarding health and controlling healthcare utilization requires effective disease management, greater education for non-specialists, rapid referral routes for diagnosis and collaborative care between specialists and non-specialists.

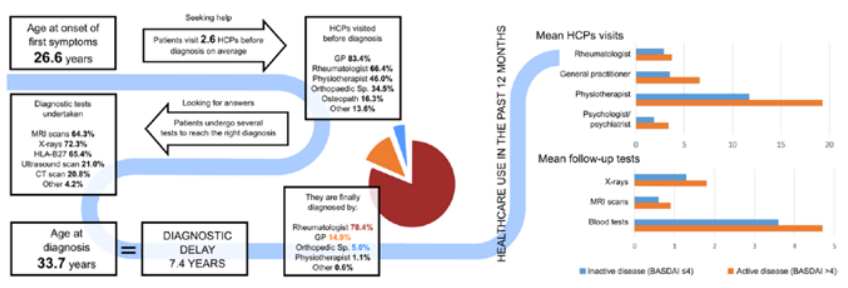

Figure 1. axSpA Patient journey according to EMAS

Acknowledgements: This study was supported by Novartis Pharma AG. The authors would like to thank all participants who participated in this study. Disclosure of Interests: Marco Garrido-Cumbrera: None declared, Denis Poddubnyy Consultant of: Abbvie, BMS, Celgene, Janssen, Lilly, MSD, Novartis, Pfizer, Roche, and UCB, Grant/research support from: Abbvie, MSD, Novartis and Pfizer, Christine Bundy Consultant of: Abbvie, Celgene, Janssen, Lilly, Novartis, and Pfizer, Laura Christen Employee of: Novartis Pharma AG, Raj Mahapatra: None declared, Souzi Makri: None declared, Carlos Jesús Delgado-Domínguez: None declared, Sergio Sanz-Gómez: None declared, Pedro Plazuelo-Ramos: None declared, Victoria Navarro-Compán Grant/research support from: Abbvie, BMS, Lilly, MSD, Novartis, Pfizer, Roche, and UCB

DOI: 10.1136/annrheumdis-2021-eular.2426

\section{POS0245 PERFORMANCE OF STANDARDIZED SCORES FOR DISEASE ASSESSMENT AND PAIN IN PATIENTS WITH SPONDYLOARTHRITIS AND FIBROMYALGIA}

X. Baraliakos ${ }^{1}$, S. Tsiami ${ }^{1}$, P. Dukatz ${ }^{1}$, M. C. Gkelaki ${ }^{1}$, U. Kiltz ${ }^{1}$, J. Braun ${ }^{1} .{ }^{1}$ RuhrUniversity Bochum, Rheumazentrum Ruhrgebiet, Herne, Germany

Background: The pathogenesis of spondyloarthritis $(\mathrm{SpA})$ including axial $\mathrm{SpA}$ (axSpA) and psoriatic arthritis (PsA) differs from fibromyalgia (FM). However, symptoms partially overlap and both patient groups suffer from pain and stiffness. In addition, SpA patients may also develop a secondary form of FM. Classification criteria for SpA and diagnostic criteria for FM are used to differentiate between these subsets. Patient reported outcomes (PRO) often generated by questionnaires are used to assess severity and other disease features.
Objectives: To study whether PROs developed for axSpA, PsA, and related physician-based information behave in a similar way in patients diagnosed with FM without an additional chronic inflammatory rheumatic disease (CIRD) as in patients with a primary diagnosis of SpA without or with secondary FM.

Methods: Patients were consecutively recruited. The main inclusion criterion was a clinical diagnosis of FM (without CIRD), axSpA or PsA (without or with secondary FM) and the indication for a treatment adaptation (escalation or change within the same class) for any reason, based on the judgement of experienced rheumatologists. Standardized assessment tools and lab parameters (BASDAI, ASDAS-CRP, DAPSA, patient's and global assessment (NRS), CRP, BASFI, Fibromyalgia Impact questionnaire (FIQ), Leeds Enthesitis Index (LEI), Maastricht Ankylosing Spondylitis (MASES) and SpA Research Consortium of Canada (SPARCC) Enthesitis Score were assessed and compared between subgroups.

Results: The baseline demographics of 300 recruited patients (100 FM. 100 axSpA and 100 PsA) are shown in Table 1. All patients with FM (primary or secondary to $\mathrm{SpA}$ ) showed the highest scores in almost all assessments, and this was independent of the main diagnosis (Table 2). In comparison, patients with axSpA or PsA without secondary FM showed significantly lower scores in al PROs as compared to those with primary and secondary FM, with exception of (i) scores of ASDAS-CRP and (ii) duration of morning stiffness (Question 6 of BASDAI), which were not affected by the presence of secondary FM (Table 2). Conclusion: Secondary FM is leading to significantly higher levels of SpA-specific scores. ASDAS-CRP was the only score that was not influenced by the presence of secondary FM in patients with axSpA even though it was also increased in patients with primary FM, while similar results were found for the duration but not the level of morning stiffness. On the other hand, FM-specific questionnaires also showed high scores in patients with axSpA and PsA with concomitant FM but not in those without.

Table 1. Baseline characteristics of all diagnosis subtypes and comparison ( $p$-values) to primary FM diagnosis. ' + ': diagnosis with concomitant FM, '-': diagnosis without concomitant FM

\begin{tabular}{|c|c|c|c|c|c|c|c|c|c|}
\hline Table 1 & FM & axSpA- & p-value & axSpA+ & p-value & PSA- & p-value & PsA+ & $p$-value \\
\hline Age & $56.4 \pm 10.2$ & $53.5 \pm 14.2$ & 0.086 & $48.7 \pm 13.5$ & 0.026 & $56.0 \pm 13.2$ & $<0.001$ & $58.5 \pm 10.4$ & 0.962 \\
\hline Male & $2 \%$ & $67 \%$ & $<0.001$ & $0 \%$ & -- & $38 \%$ & $<0.001$ & $6 \%$ & 0.322 \\
\hline $\begin{array}{c}\text { HLAB27 } \\
\text { pos. }\end{array}$ & $0 \%$ & $84 \%$ & - & $71 \%$ & - & $16 \%$ & - & $50 \%$ & - \\
\hline CRP (mg/dl) & $0.3 \pm 0.5$ & $1.1 \pm 2.1$ & 0.001 & $0.5 \pm 0.3$ & 0.035 & $1.4 \pm 3.0$ & 0.001 & $0.5 \pm 0.5$ & 0.031 \\
\hline NRS pain & $7.5 \pm 1.6$ & $6.1 \pm 2.4$ & $<0.001$ & $7.7 \pm 1.8$ & 0.768 & $6.4 \pm 2.2$ & $<0.001$ & $7.3 \pm 1.1$ & 0.223 \\
\hline
\end{tabular}

Table 2. Mean values ( \pm standard deviation) of the assessed diseasespecific indices and comparison ( $p$-values) to primary FM diagnosis.

\begin{tabular}{|c|c|c|c|c|c|c|c|c|c}
\hline Table 2 & FM & axSpA- & $p$-value & axSpA+ & p-value & PSA- & p-value & PSA+ & p-value \\
\hline BASDAI & $6.9 \pm 1.4$ & $5.2 \pm 2.0$ & $<0.001$ & $6.9 \pm 1.4$ & 0.858 & - & - & - & - \\
\hline $\begin{array}{c}\text { BASDA } \\
\text { Q1: Fatigue }\end{array}$ & $7.4 \pm 1.9$ & $5.7 \pm 2.6$ & $<0.001$ & $7.8 \pm 1.2$ & 0.667 & - & - & - & - \\
\hline ASDAS-CRP & $3.3 \pm 0.6$ & $3.1 \pm 1.0$ & 0.086 & $3.7 \pm 1.2$ & 0.208 & - & - & - & - \\
\hline BASF1 & $6.4 \pm 2.1$ & $5.4 \pm 2.5$ & 0.005 & $7.1 \pm 1.8$ & 0.41 & - & - & - & - \\
\hline DAPSA & $43.0 \pm 17.8$ & - & - & - & - & $32.0 \pm 18.6$ & $<0.001$ & $46.5 \pm 19.7$ & 0.37 \\
\hline FIQ & $68.5 \pm 13.5$ & $53.9 \pm 21.2$ & $<0.001$ & $72.3 \pm 13.7$ & 0.352 & $57.2 \pm 18.3$ & $<0.001$ & $68.5 \pm 11.6$ & 0.978 \\
\hline LEI & $4.0 \pm 1.6$ & $1.5 \pm 1.7$ & $<0.001$ & $3.3 \pm 1.4$ & 0.179 & $2.4 \pm 2.0$ & $<0.001$ & $3.6 \pm 2.0$ & 0.625 \\
\hline MASES & $8.6 \pm 3.0$ & $3.4 \pm 3.3$ & $<0.001$ & $8.2 \pm 2.9$ & 0.642 & $4.2 \pm 3.6$ & $<0.001$ & $7.1 \pm 3.6$ & 0.101 \\
\hline SPARCC & $9.4 \pm 3.4$ & $3.5 \pm 3.4$ & $<0.001$ & $7.7 \pm 3.8$ & 0.139 & $5.1 \pm 3.7$ & $<0.001$ & $8.1 \pm 3.9$ & 0.412 \\
\hline
\end{tabular}

Disclosure of Interests: None declared

DOI: 10.1136/annrheumdis-2021-eular.3139

\section{Clinical vasculitis}

\section{POS0246 \\ THE WORLDWIDE INCIDENCE AND PREVALENCE OF ANCA VASCULITIS: A SYSTEMATIC REVIEW AND META-ANALYSIS OF EPIDEMIOLOGICAL STUDIES}

R. Redondo Rodriguez ${ }^{1}$, N. Mena-Vázquez ${ }^{1}$, A. M. Cabezas-Lucena ${ }^{1}$, F. GodoyNavarrete $^{1}$, M. Morales-Águila ${ }^{1}$, S. Manrique Arija ${ }^{1} .{ }^{1}$ Instituto de Investigación 
Biomédica de Málaga. Hospital Regional Universitario de Málaga, Reumatología, Málaga, Spain

Objectives: To evaluate the worldwide incidence and prevalence of ANCA vasculitis through a systematic review of the literature and meta-analysis.

Methods: A systematic search of MEDLINE and EMBASE search engines was carried out for studies that analyzed the incidence and prevalence of ANCA vasculitis in different geographical areas. Inclusion criteria: patients diagnosed with ANCA vasculitis according to ACR criteria/ Chapel Hill Consensus and adult patients (> 16 years). All ANCA vasculitis (microscopic polyangiitis, granulomatosis with polyangiitis, eosinophilic granulomatosis with polyangiitis) were considered. Exclusion criteria: editorials, conference abstracts, case or cases series reports and narrative reviews; insufficient description of the methods; lack of data to compute incidence or prevalence; and duplicate studies.Variables: Main variable: the pooled prevalence measured by the number of prevalent cases per million / person-year $(95 \% \mathrm{Cl})$ and the pooled incidence measured as the number of incident cases per million / person-year $(95 \% \mathrm{Cl})$. Secondary variables: the prevalence and incidence of each vasculitis ANCA and according geographic area. A meta-analysis was undertaken to estimate the pooled incidence and the pooled prevalence per million / person-years. The $95 \% \mathrm{Cl}$ and $\mathrm{I} 2$ for heterogeneity were calculated. Results: Twenty four studies were included. The pooled incidence $(95 \% \mathrm{Cl})$ was 12.2 per million / person-year (8.4-16.5) and the pooled prevalence $(95 \%$ $\mathrm{Cl})$ was 130 per million / person-year (67.5-213). The individual incidence for each vasculitis was: GPA (6.7), MPA (5.9) and EGPA (1.6). The individual prevalence for each vasculitis was: GPA (69.3), MPA (21.9) and EGPA (13.5).

In the analysis by continents, the pooled incidence for GPA vasculitis was higher in Europe (7.5), while the pooled incidence for MPA vasculitis was higher in America (6.9) and for EGPA vasculitis it was higher in Asia (1.8). The pooled prevalence for GPA and MPA vasculitis was higher in Europe (83.9,24.4 respectively) than in America (14.2, 12.8 respectively).

Conclusion: The pooled incidence and the pooled prevalence are higher in the case of GPA vasculitis compared to the rest of ANCA vasculitis. In general there is a predominance of incidence and prevalence of all ANCA vasculitis in the northern hemisphere compared to the south.

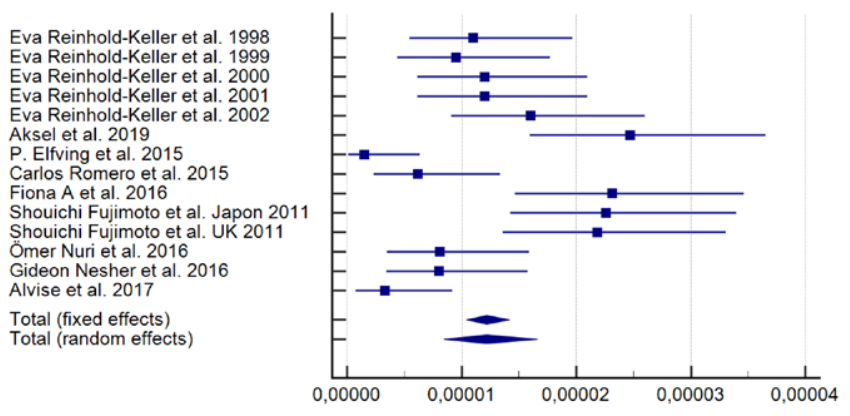

Figure 1. The pooled incidence ANCA vasculitis.

Fure 1. The pooled incidence ANCA vasculitis.

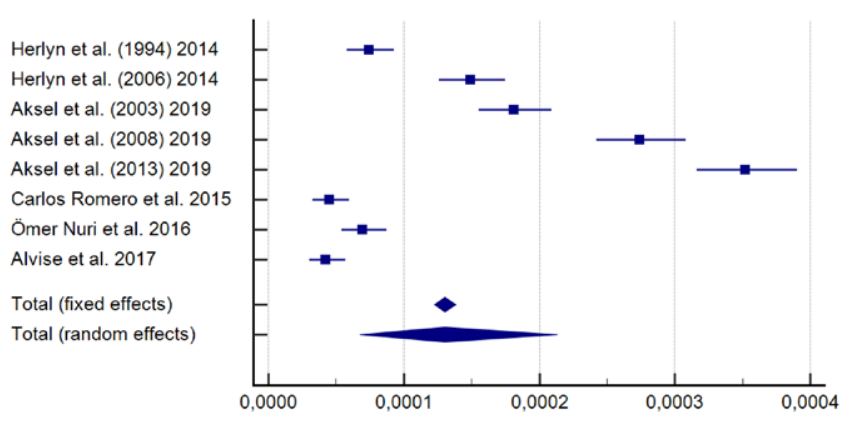

Figure 2. The pooled prevalence ANCA vasculitis

Disclosure of Interests: None declared

DOI: 10.1136/annrheumdis-2021-eular.1630

\section{POS0247 \\ INTERSTITIAL ANCA-ASSOCIATED VASCULITIS ASSOCIATES WITH SEVERE KIDNEY INJURY INDEPENDENT OF GLOMERULONEPHRITIS}

S. Hakroush ${ }^{1}$, B. Tampe ${ }^{2}{ }^{1}$ University Medical Center Göttingen, Institute of Pathology, Göttingen, Germany; ${ }^{2}$ University Medical Center Göttingen, Department of Nephrology and Rheumatology, Göttingen, Germany
Background: Antineutrophil cytoplasmic antibody (ANCA)-associated vasculitis (AAV) is a small vessel vasculitis affecting multiple organ systems, including the kidney. Small vessels in the kidney include small-sized arteries (interlobular artery, afferent and efferent arteriole), capillaries (glomerular and peritubular capillary) and venules.

Objectives: Although crescentic ANCA glomerulonephritis (GN) is a common histological finding reflecting glomerular small vessel vasculitis, it is reasonable that manifestation of AAV could also contribute to interstitial small vessel vasculitis. Therefore, we here aimed to expand our current knowledge focusing on interstitial vasculitis in ANCA GN by systematic histological scoring of vascular lesions analogous to Banff.

Methods: A total number of 49 kidney biopsies with confirmed renal involvement of AAV at the University Medical Center Göttingen were retrospectively included between 2015 till 2020. A renal pathologist (SH) evaluated all biopsies and was blinded to clinical data collection and analysis. A detailed methological section is provided in the Supplementary material and methods section.

Results: Since previous studies established that crescentic ANCA GN associates with severe kidney injury and acute deterioration of kidney function in AAV, we first systematically scored interstitial vasculitis in association with requirement of renal replacement therapy (RRT). Among all active and chronic tubulointerstitial lesions analogous to the Banff scoring system, the only association between severe kidney injury requiring RRT was observed for interstitial vasculitis in AAV reflected by peritubular capillaritis ( $p t c, p=0.0002)$ and arteritis $(v, p=0.0069)$, affecting $5 / 49(10.2 \%)$ and $11 / 49(22.4 \%)$ of renal biopsies, respectively. Since it is known that severe deterioration of kidney function also correlates with crescentic ANCA GN, we next directly compared glomerular and tubulointerstitial lesions. The fraction of normal glomeruli was inversely associated with interstitial fibrosis (ci), total (ti) and inflammation in IFTA (i-IFTA), whereas glomerular crescents were associated with interstitial inflammation (i), tubulitis (t) and total inflammation (ti). In contrast, global glomerular sclerosis associated with less interstitial inflammation (i) but correlated with interstitial fibrosis (ci) and tubular atrophy (ct), confirming established mechansim that chronic glomerular injury leads to tubular atrophy and interstitial fibrosis. Interestingly, no association between interstitial vasculitis (ptc and v correlating with severe kidney injury) and any glomerular lesion in ANCA GN (also correlating with severe kidney injury) was observed, thereby confirming that interstitial vasculitis contributes to severe kidney injury independent of ANCA GN. By contrast, short-term renal recovery from RRT was equal in both groups, suggesting a distinct association with acute decline of kidney function at disease onset.

Conclusion: Taken together, by using the Banff scoring system we here expand our current knowledge of renal interstitial lesions in AAV revealing peritubular capillaritis and arteritis as important histological alterations associated with severe kidney injury in a considerable subset of AAV. Furthermore, our findings that interstitial vasculitis did not correlate with crescentic ANCA GN implicate that the characteristics of each vasculitis manifestation are independent and could further improve our understanding of mechanisms contributing to renal injury. These observations suggest that interstitial vasculitis in AAV may also affect long-term prognosis requiring further investigation.

Disclosure of Interests: None declared

DOI: 10.1136/annrheumdis-2021-eular.4296

\begin{tabular}{|l|}
\hline POS0248 \\
PSYCHOMETRIC PROPERTIES OF OUTCOME \\
MEASUREMENT INSTRUMENTS FOR ANCA- \\
ASSOCIATED VASCULITIS: A SYSTEMATIC \\
LITERATURE REVIEW
\end{tabular}

A. Berti ${ }^{1,2}$, G. Boleto ${ }^{3}$, P. A. Merkel ${ }^{4}$, G. Tomasson ${ }^{5}$, S. Monti ${ }^{6}$, K. A. Quinn ${ }^{7}$, L. Carmona ${ }^{8}$, S. Ramiro ${ }^{9,10}$ on behalf of OMERACT Vasculitis Working Group. ${ }^{1}$ Hospital of Trento, University of Trento, Rheumatology, Trento, Italy; ${ }^{2}$ Mayo Clinic, Thoracic Disease Research Unit, Rochester, United States of America; ${ }^{3}$ Cochin Hospital, Rheumatology, Paris, France; ${ }^{4}$ Penn Medicine, University of Pennsylvania Health System, Rheumatology, Philadelphia, United States of America; ${ }^{5}$ Landspitali The National University Hospital of Iceland, Rheumatology, Reykjavik, Iceland; ${ }^{6}$ University of Pavia, Rheumatology, Pavia, Italy; ${ }^{7}$ National institute of arthritis and musculoskeletal and skin diseases, Rheumatology, Bethesda, United States of America: ${ }^{8}$ Instituto de Salud Musculoesquelética (INMUSC), Rheumatology, Madrid, Spain; ${ }^{9}$ Department of Rheumatology, Leiden University Medical Center, Rheumatology, Leiden, Netherlands; ${ }^{10}$ Department of Rheumatology, Zuyderland Medical Center, Rheumatology, Heerlen, Netherlands

Background: The OMERACT Vasculitis Working Group has defined a Core Domain Set of outcome measures for ANCA-associated vasculitis (AAV). However, the psychometric properties of available outcome measurement instruments in AAV, an essential consideration when choosing among instruments, have not been summarized.

Objectives: To systematically review and summarize the psychometric properties of outcome measurement instruments used in AAV. 